\title{
THE EFFECT OF TILT ANGLE ON HEAT TRANSFER IN
} CIRCULAR PIPE

\author{
Eldesouki I. Eid ${ }^{\mathrm{a}}$, Mohamed A. Teamah ${ }^{\mathrm{b}}$, Mohamed M. Khairat Dawood ${ }^{\mathrm{c}}$, Reda \\ A.Khalfallaha, Mohamed A. Abd Elgaleel Elsedy ${ }^{a}$ \\ ${ }^{a}$ Mechanical Dept,Faculty of Industrial Education, Suez University, \\ Egypt,+201227835110,eldesukieid@yahoo.com \\ ${ }^{\mathbf{b}}$ Mechanical Eng. Dept, Faculty of Engineering, Alexandria University \\ Egypt,+201003101997,mteamah@yahoo.com \\ ${ }^{c}$ MechanicalDept, Faculty of Engineering, Suez Canal \\ UniversityEgypt,+201099558136,khairat4975@yahoo.com \\ ${ }^{a}$ Mechanical Dept,Faculty of Industrial Education, Suez University \\ Egypt,+201227460887,Reda.Khalafalla@suezuniv.edu.eg \\ ${ }^{a}$ Mechanical Dept,Faculty of Industrial Education, Suez University \\ Egypt,+201226887601,mahamedabdelgaleel@yahoo.com
}

This paper study of the effect of tilt angle on heat transfer in circular pipe from an inclination copper pipe, $0^{\circ}$ to $90^{\circ}$ made $500 \mathrm{~mm}$ length, $50 \mathrm{~mm}$ diameter. experiments are conducted for a wide range of Richardson numbers $0.05<\mathrm{Ri}<1$. The Reynolds, $1581.139<$ $\operatorname{Re}<25641.4$ the study is used of a wide range of Richardson and Reynolds numbers covering the forced convection dominant and mixed convection regimes. In order to quantify the enhancement of the heat transfer changing tilt angle, The best rate of heat transfer was achieved at $0^{\circ}$ of the copper. The effect of the tilt angle on heat transfer was studied and the pressure drop, so we studied the best ratios filled with the least pressure drop, the outlet velocity increase the rate of heat transfer at the lowest cost and can be used in many fields and industrial applications.

Keywords:tilt angle,mixed convection, circular pipe.

Corresponding Author:(Mohamed A. AbdElgaleelElsedy)mahamedabdelgaleel@yahoo.com

\section{INTRODUCTION}

Heat transfer enhancement techniques can be classified in two main groups, i.e. active and passive methods. Active methods enhance heat transfer greatly but with added cost to the process such as adding mechanical energy or increasing thermal capacity of the medium of

\begin{tabular}{|c|c|c|}
\hline \multicolumn{3}{|c|}{ NOMENCLATURE } \\
\hline A & Area, $\mathrm{m}^{2}$ & $\underline{\text { Dimensionless }}$ \\
\hline $\mathrm{cp}$ & Specific heat capacity, $\mathrm{J} / \mathrm{kg} \mathrm{K}$ & $\overline{G r} \quad$ Grashof number, $\left(\mathrm{g} \beta \mathrm{q}^{\prime \prime} \mathrm{D}^{4} / v^{2} \mathrm{k}\right)$ \\
\hline $\mathrm{D}$ & Inner diameter of the pipe, $\mathrm{m}$ & Nusselt number, (hd/k) \\
\hline$g$ & Gravity acceleration, $\mathrm{m} / \mathrm{s}^{2}$ & Reynolds number, $(\mathrm{Ud} / \mathrm{v})$ \\
\hline $\mathrm{h}$ & Heat transfer coefficient, $\mathrm{W} / \mathrm{m}^{2} \mathrm{~K}$ & Richardson number, $\left(\mathrm{Gr} / \mathrm{Re}^{2}\right)$ \\
\hline $\mathrm{k}$ & Thermal conductivity, W/m K & Greek symbols \\
\hline $\mathrm{m}^{\circ}$ & Mass flow rate $(\mathrm{kg} / \mathrm{s})$ & $\beta \quad$ Thermal expansion coefficient, $(1 / \mathrm{K})$ \\
\hline$q^{\prime \prime}$ & Heat flux, $\left(\mathrm{W} / \mathrm{m}^{2}\right)$ & \\
\hline & & o Kinematic viscosity, $\mathrm{m}^{2} / \mathrm{s}$ \\
\hline $\mathrm{t}$ & Temperature, ${ }^{\circ} \mathrm{C}$ & $\underline{\text { Subscrip }}$ \\
\hline$\Delta \mathrm{T}$ & Temperature difference, $\left[\mathrm{t}_{\mathrm{s}}-\mathrm{t}_{\mathrm{f}}\right],{ }^{\circ} \mathrm{c}$ & Wall pipe surface \\
\hline $\mathrm{U}$ & Velocity, $\mathrm{m} / \mathrm{s}$ & average \\
\hline
\end{tabular}


heat exchange. Passive methods, on the other hand, enhance heat transfer without adding cost to the process. One of the passive techniques is the use of which is an area of interest for many researchers. theenhances heat transfer by reducing boundary layer thickness and slowing down the cross flow.Nanotechnology was used to improve heat transfer in many industrial fields with the use of this technique and obtained high efficiency results. This is shown in previous studiesKunugi et al [1] Ultrahigh heat transfer enhancement using copper oxide, aluminium oxide and carbon nano particles tube as water working fluid, the experimental and numerical study depend on nano, axis and Reynolds number parameters can extremely enhance the heat transfer and heat exchange performance.Nazari et al [2] Experimental study of convective heat transfer using nano fluids $\mathrm{Al}_{2} \mathrm{O}$ /Water througha pipe filled, The volume fractions of $\mathrm{Al}_{2} \mathrm{O}_{3}$ nanofluids are selected as $0.1,0.25,0.5,1$, and $1.5 \%$. The maximum increment in the Nusslet number is about $57 \%$ at $\mathrm{Re}=3704$ inside the tube filled.The obtained result showed maximum increase of $39 \%$ in the pressure drop for (with volume fraction of $1.5 \%$ ) at $\mathrm{Re}=3704 . \mathrm{Xu}$ et al [3] Review on heat conduction, heat convection, thermal radiation and phase change heat transfer of nanoparticles with shear flow and fouling media: Fundamentals and applications depended on three methods. Flow and thermal transport of fluids, Transport of nanofluids and nanoparticles and The nanofluid transport studies. The enhancing boiling heat transfer, anti-freezing, increasing the heat transfer rate in thermal storage process. There are several previous numerical and experimental studies. The cylindrical tubes and different sections are displayed as follows.Rashidi et al [4] Fluid flow and forced convection heat transfer around a solid cylinder wrapped with a homogenous .Numerical analysis and clear fluid region. The Porous layer thickness permeability and Reynolds number parameters study. The convection heat transfer coefficient increases with increase in Darcy number with low thermal conductivity.Akansu [5] Numerical Analysis heat transfers and pressure drops for turbulators in a circular air pipe. The Reynolds number, the blockage ratio, and height ratio parameters. The result showed increasing the near velocity of the pipe walls with increasing blockage ratio, the higher heat-transfer enhancement was obtained in the case of L/D and H/D being 0.5 and 0.2 respectively. Kaya [6] Effects of buoyancy and conjugate heat transfer on nonDarcy mixed convection about a vertical slender hollow cylinder embedded, the Richardson number parameters achieved An increase in the parameter $\mathrm{k}_{1}$ and Forcheimer parameter $\mathrm{F}^{*}$, increases the local skin friction and local heat transfer parameters and decreases the dimensionless interfacial temperature distributions for low values of stream wise direction.Nazaret al [7] The Brinkman model for the mixed convection boundary layer flow past a horizontal circular cylinder in medium. The mixed convection parameter achieved an increase in the value of $\Gamma$ leads to a decrease of the wall heat transfer and the skin friction coefficient. Braga and Lemos [8] Simulation of turbulent natural convection cylindrical annulus using a macroscopic two-equation model. Al-Sumaily and Thompson [9] Forced convection from a circular cylinder in pulsating flow with and without the presence of nonmetallic materials. The numerical result showed much higher heat transfer enhancement from the cylinder than that promoted by using pulsating flow is laminar and incompressible based oscillating frequency parameter. Hutter et al [10] Heat transfer in metal foams and experimental designed copper metal form filled tubular reactors. The result compared to theempty tube case the foam inserts lead to an increase in heat transfer, which is up to 10 times higher. Rong et al [11] Numerical study of heat transfer enhancement in a pipe by axisymmetric lattice Boltzmann model based on Graphics Processing Unit, the layer thickness parameters study Achievedcan effectively improve heat transfer, the flow resistance also increases. It also suggests that enhancing heat transfer and reducing flow resistance are contradictory, to achieve a optimal thermal performance in practice.Corasaniti and Gori [12] Natural convection around a vertical cylinder (thermal probe) immersed In glass beads with 
particles of the same diameter a medium and is saturated with water, the heat transfer coefficient is very good agreement with the theoretical evaluation with change Probe length and axial coordinate. Tian et al [13] Experimental study on flow and heat transfer of power law fluid in structured Spheres packed particles with The Partially Hydrolyzed Polyacrylamide (HPAM) solutions, the result showed the increasing of Reynolds numbers and concentration of HPAM, the integrated heat transfer coefficient gradually decreased, the findings would be useful for the optimal design of packed beds. Also the temperature difference between particle and fluid along the flow direction was analyzed.Mancin et al [14] Experimental results and modeling an open-circuit type wind tunnel with a rectangular cross section, the important parameters achieved heat transfer coefficient and the foam finned surface efficiency in order to optimize the overall heat transfer performance for a given foam structure. Kurtbas and Celik[15] Experimental investigation of forced and mixed convection heat transfer in filled horizontal rectangular channel, Average Nusselt number increases proportional, in a horizontal rectangular channel which is filled. Hamadouche et al [16] Experimental investigation of convective heat transfer in solid blocks foams technique of air cooling electronic systems. Height, width of open cell foam and solid blocks is parameters. The result showed the foam inserts in horizontal rectangularchannel, an optimum sample height was found around $1.0 \mathrm{H}$, for the range of the considered parameters, which can be used to enhance the heat transfer in heatexchangers. Kim et al [17] Forced convection from aluminum materials in an asymmetrically heated channel. Darcy number and Surface area to volume ratio is parameters study, Experimental results indicate that the friction factor is much higher at the lower foams while the significant enhancement in $\mathrm{Nu}$ is obtained. Kamath et al [18] Experimental investigation of flow assisted mixed convection in vertical air channels, Correlations for the Nusselt number and enhancement ratio are developed. with change are seen to enhance heat transfer in all cases though they come with a small penalty of increased pressure drop.

\section{Experimental setup}

The device consists of various main components, a $50 \mathrm{~cm}$ long copper pipe, a diameter of 5 $\mathrm{cm}$, mounted on a holder to control the inclination angle of the pipe. 50 thermocouples were installed on the surface of the pipe on 6 sections of each section 8 thermocouples, the distance between each section and the other $10 \mathrm{~cm}$. The main heater was installed on the surface of the pipe, about 17 roles, between the role and the other about $2 \mathrm{~cm}$, the heater was insulated with laminate and glass wool. The other heater was installed to compensate the heat losses and was then isolated again with laminate and glass wool (see Fig. 1). Control the air velocity by blower air of various velocities connected to the flexible joint with the pipe to enter the air. The pipe was fitted with two openings to enter and exit the air to measure the pressure difference.

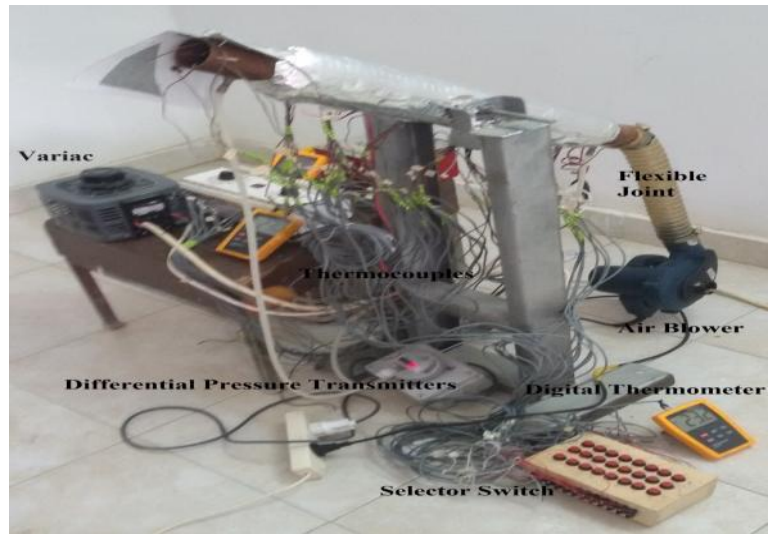

Fig 1: Photograph of the experimental setup 


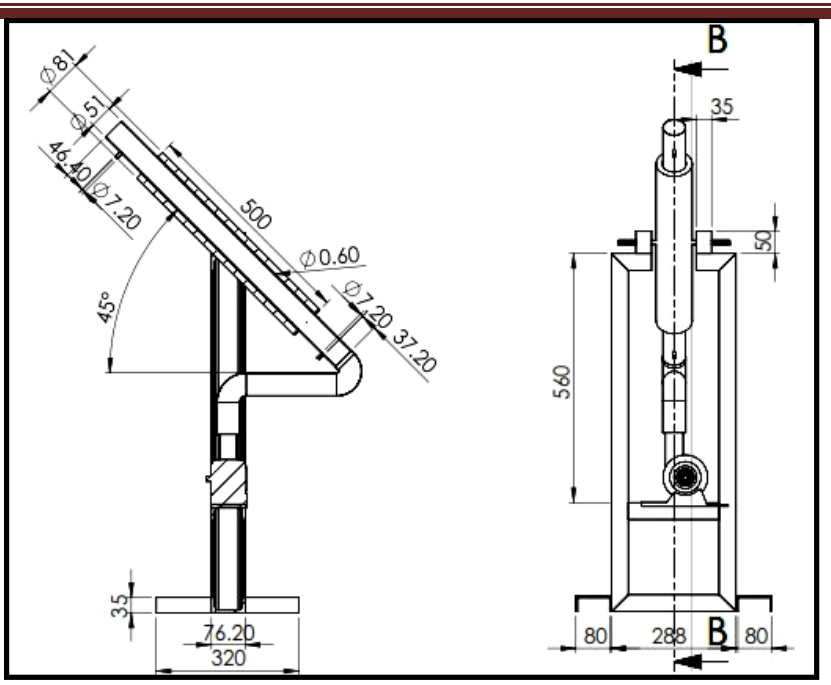

Fig. 2. Schematic diagram of the test section

Ambient temperature is measured by a $\mathrm{K}$ type thermocouple located outside axial fan of the inlet of the cylinder. To account for heat loss two thermocouples are located between the main and guard heater. Also measured the inlet and out temperature of air entry the tube section. All the thermocouples are connected to selector switch measured by Digital thermometer (model DM $6801 \mathrm{~A}^{+}$). An electrically heated element is inserted through the center to generate a constant heat flux to heat the cylinder. The constant power input to the heater is controlledusing VARIAC power controller, single phase, 50, HZ, rated capacity 1 $\mathrm{KVA}$, which has a voltage range of $0-250 \mathrm{~V}$ and a current range of $0-1.5 \mathrm{~A}$. The velocity of the air at the inlet and outlet of the test section is measured by an anemometer (model 8330 air). The pressure drop between the upstream and downstream of the cylinder assembly is measured by boring pressure taps on the cork cylinder located at a distance of $600 \mathrm{~mm}$ between Inlet and outlet pipe as shown in Fig. 2(a). The pressure drop across the pipe measured by differential pressure transmitterswith digital display(model DBTM250D). The air inlet velocities of $0.5-8 \mathrm{~m} / \mathrm{s}$. Air at atmospheric temperature of 29 to $33^{\circ} \mathrm{C}$ is blown into the test section by an axial fan located at the bottom of the cylinder. The pressure drop across measured with a digital pressure manometer. The velocity and the pressure drop across the foam are recorded.

Heat transfer experiments are conducted for different flow rates and heat input. The procedure for conducting experiments is as follows.

The axial fan is switched $\mathrm{ON}$ and the required rpm is set on the cylinder of the variable frequency AC drive. The DC power supply is switched on and the voltage is adjusted in order to obtain the desired the constant heat input. When the Not a difference in thermocouple reading is between main and guard heater andthe surface temperature of the tube is stabilized and this is achieved after approximately 150 minutes, it is assumed that steady state has been reached and the reading data recorded. The heater voltage and current, the inlet air velocity and the pressure drop across the pipe are measured and recorded. The power of the heater is varied from $715 \mathrm{~W} / \mathrm{m}^{2}$. The fan air flow velocities varied from 0.5 to $7 \mathrm{~m} / \mathrm{s}$ The mass flow rate of air is varied from $0.117-1.884 \mathrm{~kg} / \mathrm{s}$ and the pressure varies within $0-700 \mathrm{~Pa}$ range. By a simple conduction heat transfer analysis across the heat loss through the Glass wool insulation is calculated.The heat loss obtained is then deducted from the heat input to the heater for the calculation of the heat transfer coefficient. The heat loss is found to exponentially decrease from $14 \%$ to $0.8 \%$ with an increase in the Reynolds number, and is accounted for the heat transfer coefficient, as well as for all the correlations developed. 


\section{Experimental uncertainty analysis}

The aim of this study is to study the effect of tilt angle on heat transfer on a wall of a cylinder. This is achieved by conducting various practical experiments with the stability of the amount of heat entering the cylinder and changing the tilt angles of the cylinder and also changing the air velocity of the cylinder.

The electrical power input, the axis ratio and the orientation angle were the parameters that effected the heat transfer rates. The uncertainty in the data calculation was based on ANSI/ASME [19]. The accuracy of the experimental study can be affected by the errors which may arise during the experiments for different reasons. The uncertainty calculation method used involves calculating derivatives of the desired variable with respect to individual experimental quantities and applying known uncertainties. The independent variables that cause errors in experiments are; input electrical power, surface area of cylinders, local cylinder surface temperatures and the ambient temperature. The magnitude of the uncertainty is estimated from Eq. (1) and maximum values of uncertainties are listed in Table 1. The maximum error for the average Nusselt,pressure drop and outlet velocity were found to be $\pm 1.5 \%, \pm 5.5 \%$ and $\pm 5.8 \%$ respectively.

$$
w_{R}=\sqrt{\left(\frac{\partial R}{\partial x_{1}} w_{1}\right)^{2}+\left(\frac{\partial R}{\partial x_{2}} w_{2}\right)^{2}+\ldots \ldots+\left(\frac{\partial R}{\partial x_{n}} w_{n}\right)^{2}}
$$

Table 1.The accuracies of the measured parameters

\begin{tabular}{|c|c|c|}
\hline \multirow{2}{*}{ Parameter } & Device & Accuracy \\
\hline Temperature & K-type thermocouple & $0.1^{\circ} \mathrm{C}$ \\
Voltage & voltmeter & $0.01 \mathrm{~V}$ \\
Current & Current meter & $0.02 \mathrm{Amp}$ \\
Pressure drop & Differential pressure transmitter & $0.02 \mathrm{~Pa}$ \\
Air velocity & Hot wire anemometer & $0.03 \mathrm{~m} / \mathrm{s}$ \\
\hline
\end{tabular}

\section{Result and Discussion}

\section{The Effect of Reynolds Numberat $0^{\circ}$}

The Nusselt number based on the hydraulic diameter is defined as.

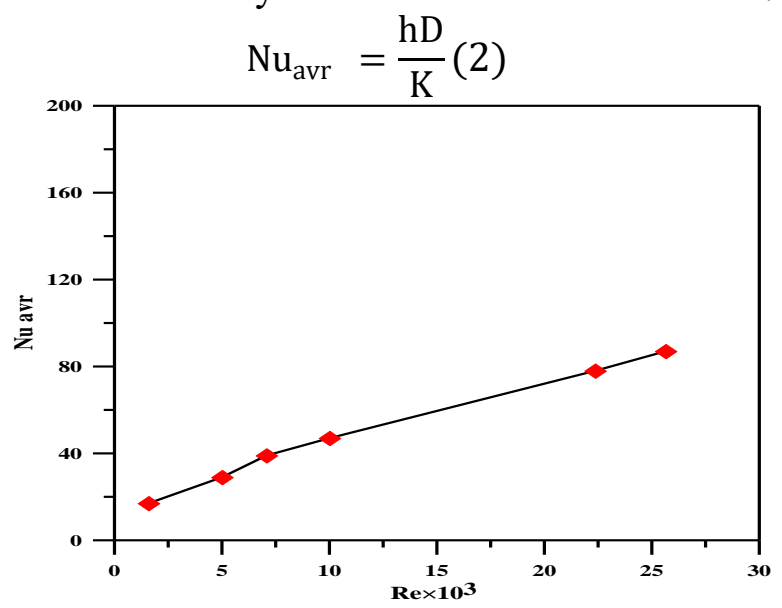

Fig 3.The Effect of Reynolds Number on the Average Nusselt Number at, $\Psi=0^{\circ}$. 
Figure (3) represents the relation between the average Nusselt number and the Reynolds number $\Psi=0^{\circ}$ at constant heat flux q" $=715 \mathrm{~W} / \mathrm{m}^{2}$. From the analysis of this figure it can be concluded that there is power relationship clear pipe between the Nusselt number and the Reynolds number. It is noticed the increasing Reynolds number increases the Nusselt number. The decreasing tube surface temperature increased the turbulent forced convection and decreases the bulk temperature at the high air velocity. The maximum fully developed Nusselt number $\mathrm{Nu}=96$ is obtained at $\mathrm{Re}=25.64 \times 10^{3}$.

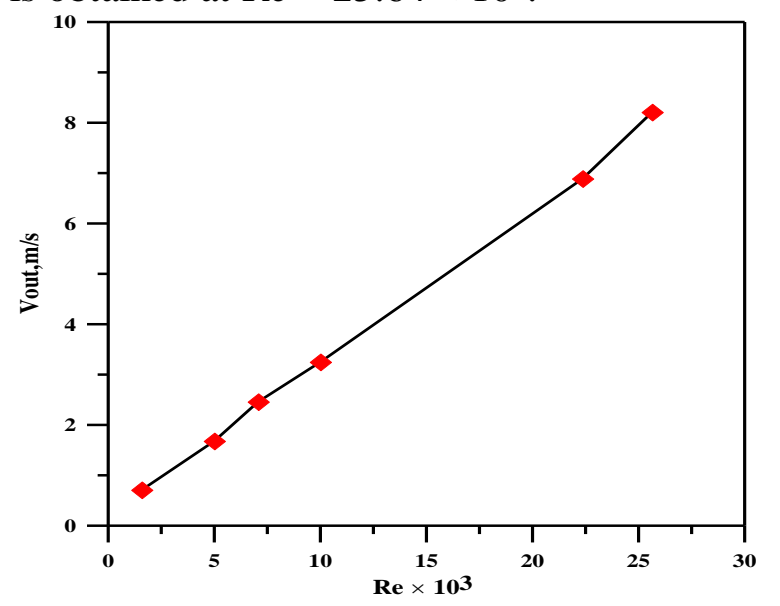

Fig 4. The Effect of Reynolds Number on outlet velocity $\Psi=0^{\circ}$

Figure (4) shows the relation between the outlet velocity and the Reynolds number at $\Psi=0^{\circ}$ at constant heat flux $715 \mathrm{~W} / \mathrm{m}^{2}$. From this figure it can be noticed that the increasing Reynolds number increases the outlet velocity. Also there is a linear relationship between the two variables. The minimum axial velocity increases value by $42 \%$ at the laminar mixed convection, the outlet minimum velocity increasing due to the air density variation is caused by the heated surface cylinder. The high velocity increasing the value by $2.6 \%$ caused by decreases the average surface temperature cylinder.

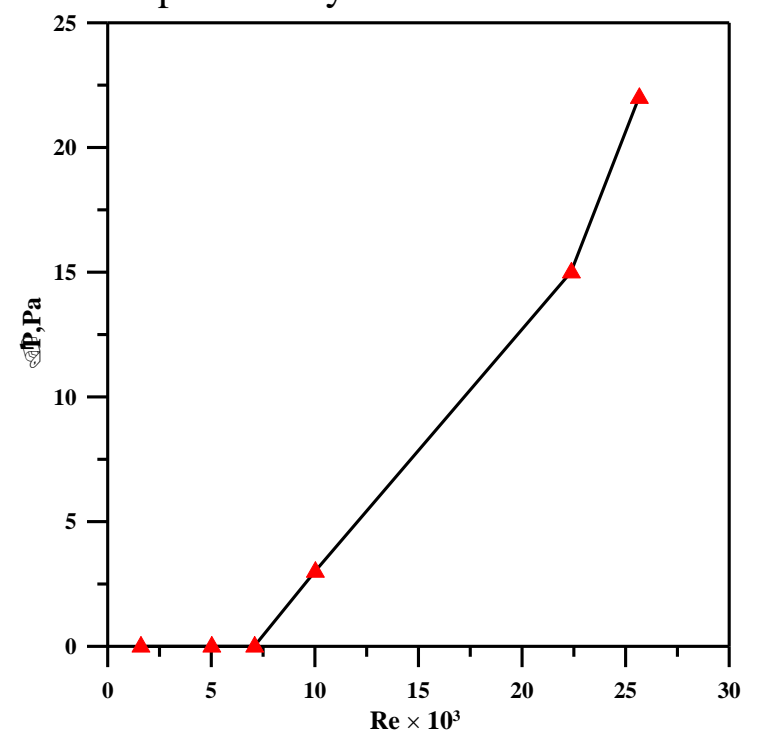

Fig 5.The Effect of Reynolds Number on The pressure drop $\Psi=45^{\circ}$

Figure (5) shows the relation between the pressure drop and the Reynolds number $\Psi=0^{\circ}$ from this figure its clear pipe. Noticed increasing The Reynolds number increases the pressure drop. Also there is a relationship between Reynolds Number and pressure drop is increasing because friction factor between the fluid and the inner surface cylinder increases, 
therefore higher velocity gradient at the wall, consequently higher drage force at the wall and higher pressure drop.

The Reynolds number, Richardson number and The Grashof number are defined based on the hydraulic diameter of the cylinder and are given as.

$$
\begin{gathered}
\mathrm{Re}=\frac{\mathrm{VD}}{\mathrm{v}} \\
\mathrm{Ri}=\frac{\mathrm{Gr}}{\mathrm{Re}^{2}} \\
\mathrm{Gr}=\frac{\mathrm{g} \beta \mathrm{q}^{\prime \prime} \mathrm{D}^{4}}{v^{2} K}
\end{gathered}
$$

The Effect of Reynolds Numberat $45^{\circ}$

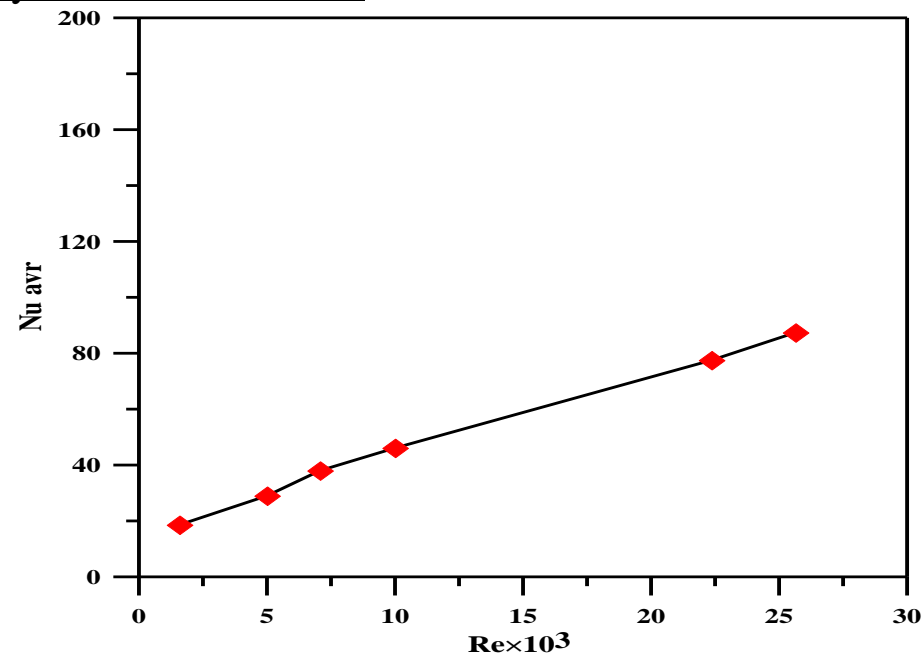

Fig 6.The Effect of Reynolds Number on the Average Nusselt Number at $\Psi=45^{\circ}$.

Figure (6) represents the relation between the average Nusselt number and the Reynolds number at $\Psi=45^{\circ}$ at constant heat flux $\mathrm{q}^{\prime \prime}=715 \mathrm{~W} / \mathrm{m}^{2}$. From the analysis of this figure it can be concluded that there is power relationship clear pipe between the Nusselt number and the Reynolds number. It is noticed the increasing Reynolds number increases the Nusselt number. The decreasing tube surface temperature increased the turbulent forced convection and decreases the bulk temperature at the high air velocity. The maximum fully developed Nusselt number $\mathrm{Nu}=92$ is obtained at $\mathrm{Re}=25.64 \times 10^{3}$.

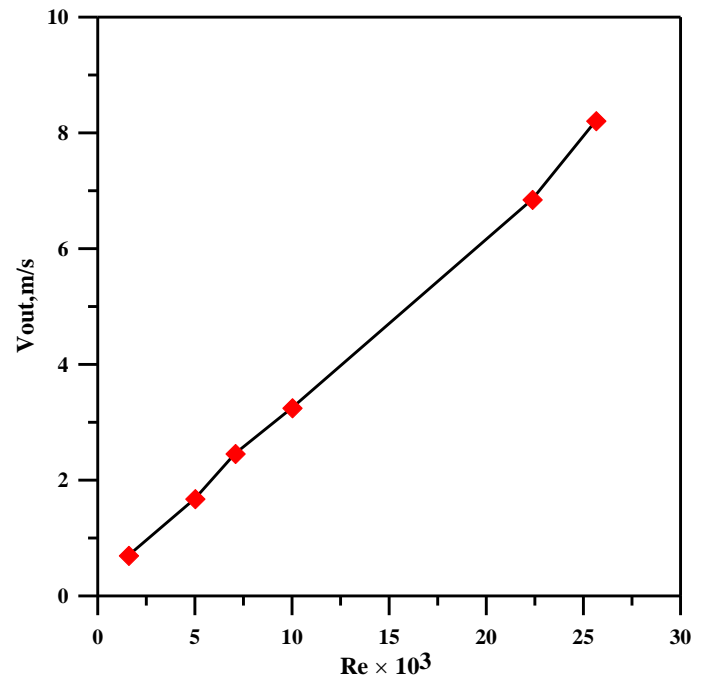

Fig 7. The Effect of Reynolds Number on outlet velocity $\Psi=45^{\circ}$ 
Figure (7) shows the relation between the outlet velocity and the Reynolds number at $\Psi=45^{\circ}$ at constant heat flux $715 \mathrm{~W} / \mathrm{m}^{2}$. From this figure it can be noticed that the increasing Reynolds number increases the outlet velocity. Also there is a linear relationship between the two variables. The minimum axial velocity increases value by $41 \%$ at the laminar mixed convection, the outlet minimum velocity increasing due to the air density variation is caused by the heated surface cylinder. The high velocity increasing the value by $2.4 \%$ caused by decreases the average surface temperature cylinder.

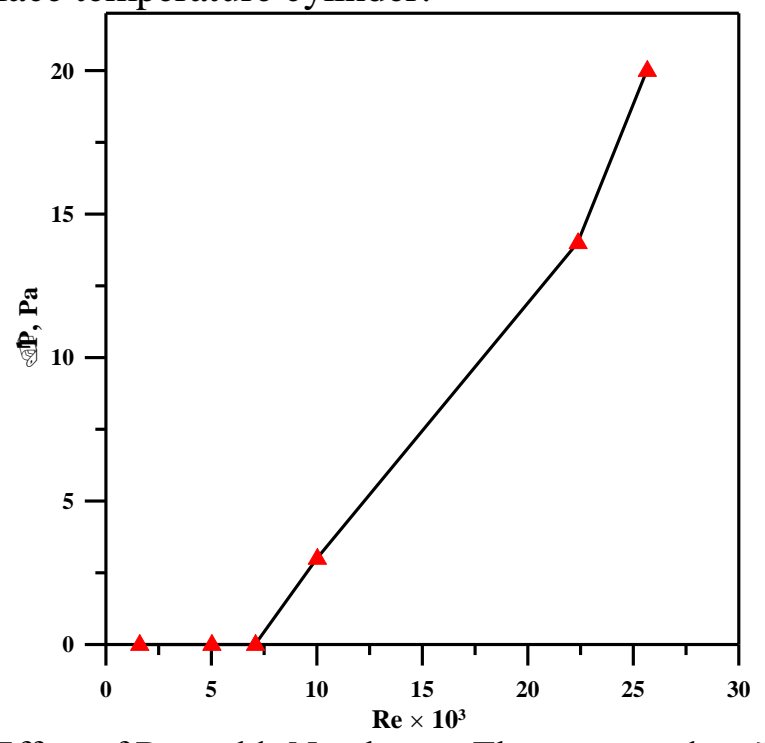

Fig 8.The Effect of Reynolds Number on The pressure drop $\Psi=45^{\circ}$

Figure (8) shows the relation between the pressure drop and the Reynolds number $\Psi=45^{\circ}$ from this figure its clear pipe. Noticed increasing The Reynolds number increases the pressure drop. Also there is a relationship between Reynolds Number and pressure drop is increasing because friction factor between the fluid and the inner surface cylinder increases the tilt angle, therefore higher velocity gradient at the wall, consequently higher drage force at the wall and higher pressure drop.

The Effect of Reynolds Numberat $90^{\circ}$

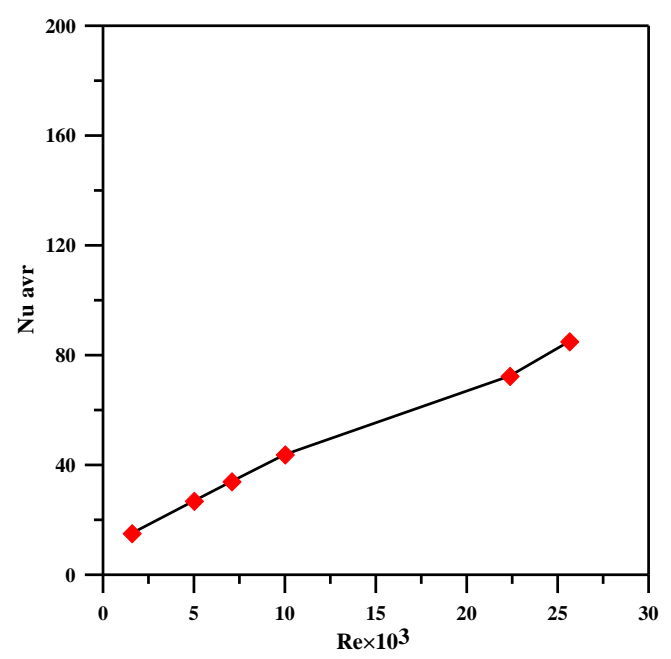

Fig 9.The Effect of Reynolds Number on the Average Nusselt Number at $\Psi=90^{\circ}$.

Figure (9) represents the relation between the average Nusselt number and the Reynolds number at $\Psi=90^{\circ}$ at constant heat flux $\mathrm{q}^{\prime \prime}=715 \mathrm{~W} / \mathrm{m}^{2}$. From the analysis of this figure it can be concluded that there is power relationship clear pipe between the Nusselt number and the 
Reynolds number. It is noticed the increasing Reynolds number increases the Nusselt number. The decreasing tube surface temperature increased the turbulent forced convection and decreases the bulk temperature at the high air velocity. The maximum fully developed Nusselt number $\mathrm{Nu}=92$ is obtained at $\mathrm{Re}=25.64 \times 10^{3}$.

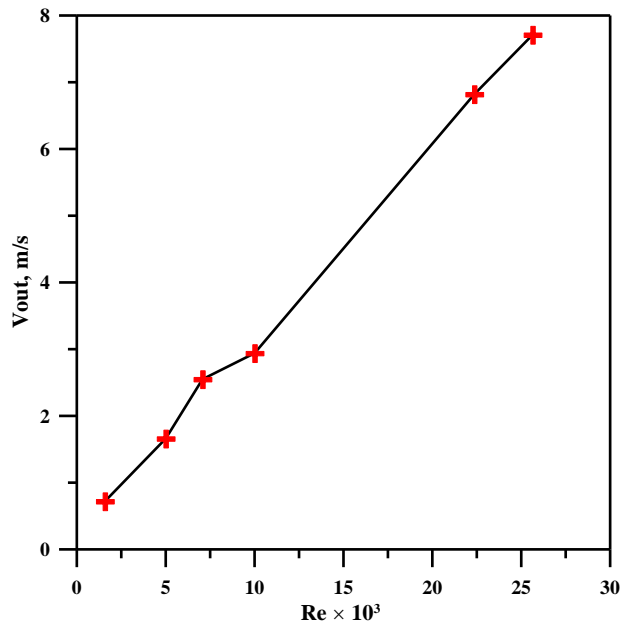

Fig 10. The Effect of Reynolds Number on outlet velocity $\Psi=90^{\circ}$

Figure (10) shows the relation between the outlet velocity and the Reynolds number at $\Psi=$ $90^{\circ}$ at constant heat flux $715 \mathrm{~W} / \mathrm{m}^{2}$. From this figure it can be noticed that the increasing Reynolds number increases the outlet velocity. Also there is a linear relationship between the two variables. The minimum axial velocity increases value by $41.1 \%$ at the laminar mixed convection, the outlet minimum velocity increasing due to the air density variation is caused by the heated surface cylinder. The high velocity increasing the value by $2.3 \%$ caused by decreases the average surface temperature cylinder.

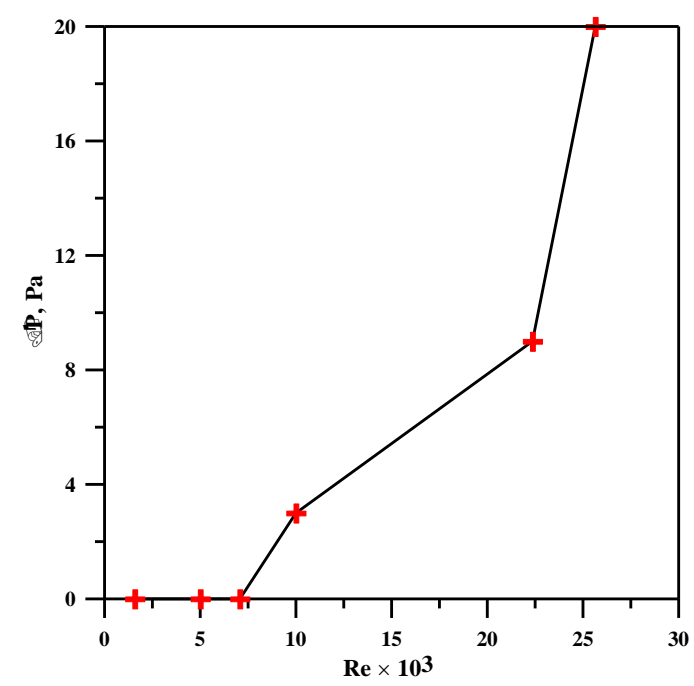

Fig 11.The Effect of Reynolds Number on The pressure drop $\Psi=90^{\circ}$

Figure (11) shows the relation between the pressure drop and the Reynolds number $\Psi=90^{\circ}$ from this figure its clear pipe. Noticed increasing The Reynolds number increases the pressure drop. Also there is a relationship between Reynolds Number and pressure drop is increasing because friction factor between the fluid and the inner surface cylinder increases, therefore higher velocity gradient at the wall, consequently higher drage force at the wall and higher pressure drop. 


\section{Conclusions}

An experimental study of heat transfer in flow mixed convection on copper metal foams of high porosity has been conducted. The results of the experiments show that for the air velocity 0.5 to $8 \mathrm{~m} / \mathrm{s}$, the heat transfer coefficient can be represented, Richardson number, and Reynolds number for the range of values used in the study. Based on the experiments, the flow regimes can be delineated into.

1. Increases the air velocityincreasing of heat transfer

2. Increases the heat transfer rate of the horizontal pipe $500 \mathrm{~mm}$

3. The forced heat transfer is dominated.

The pressure drop increasing the air velocity

\section{REFERENCES}

1) TomoakiKunugi, Katsumi Mukoaand Masahiko Shibahara, "Ultrahigh heat transfer enhancement using nano- layer", Superlattices and Microstructures 35 (2004) 531-542.

2) MohsenNazari, MojtabaAshouri, Mohammad HasanKayhani and Ali Tamayol" Experimental study of convective heat transfer of a nanofluid through a pipe filled with foam", International Journal of Thermal Sciences 88 (2015) 33-39.

3) H.J. Xu, Z.B. Xing, F.Q. Wang and Z.M. Cheng" Review on heat conduction, heat convection, thermal radiation and phase change heat transfer of nanofluids: Fundamentals and applications", Chemical Engineering Science xxx (2018) xxx-xxx.

4) SamanRashidi, Ali Tamayol, Mohammad SadeghValipour and NimaShokri" Fluid flow and forced convection heat transfer around a solid cylinder ", International Journal of Heat and Mass Transfer 63 (2013) 91-100.

5) SelahaddinOrhanAkansu."Heat transfers and pressure drops for turbulators in a circular pipe", Applied Energy 83 (2006) 280-298.

6) AhmetKaya."Effects of buoyancy and conjugate heat transfer on non-Darcy mixed convection about a vertical slender hollow cylinder embedded ", International Journal of Heat and Mass Transfer 54 (2011) 818-825.

7) RoslindaNazar, Norsarahaida Amin, Diana Filip and Ioan Pop, "The Brinkman model for the mixed convection boundary layer flow past a horizontal circular cylinder", International Journal of Heat and Mass Transfer 46 (2003) 3167-3178.

8) Edimilson J. Braga and Marcelo J.S. de Lemos, "Simulation of turbulent natural convection in cylindrical annulus using a macroscopic two-equation model", International Journal of Heat and Mass Transfer 49 (2006) 4340-4351.

9) Gazy F. Al-Sumaily and Mark C. Thompson, "Forced convection from a circular cylinder in pulsating flow with and without the presence ", InternationalJournal of Heat and Mass Transfer 61 (2013) 226-244.

10) C. Hutter , D. B uchi, V. Zuber and Ph. Rudolf von Rohr, "Heat transfer in foams", Chemical Engineering Science 66 (2011) 3806-3814. 
11) FumeiRong, Wenhuan Zhang, Baochang Shi and ZhaoliGuo, "Numerical study of heat transfer enhancement in a pipe by axisymmetric TLB model based on GPU", International Journal of Heat and Mass Transfer 70 (2014) 1040-1049.

12) SandraCorasaniti and Fabio Gori"Natural convection around a vertical cylinder (thermal probe) immersed in medium", International Communications in Heat and Mass Transfer 81 (2017) 72-78.

13) Xing-Wang Tian, Shi-Ming Xu, Zhen-Hua Sun , Ping Wang, Lin Xu and Zhang Zhang, " Experimental study on flow and heat transfer of power law fluid in structured packed ", Experimental Thermal and Fluid Science 90 (2018) 37-47.

14) Simone Mancin, Claudio Zilio, Andrea Diani and Luisa Rossetto, "Air forced convection through metal: Experimental results and modeling", International Journal of Heat and Mass Transfer 62 (2013) 112-123.

15) IrfanKurtbas and NevinCelik," Experimental investigation of forced and mixed convection heat transfer in a horizontal rectangular channel", International Journal of Heat and Mass Transfer 52 (2009) 1313-1325.

16) A. Hamadouche, R. Nebbali, H. Benahmed, A. Kouidri and A. Bousri, " Experimental investigation of convective heat transfer ", Experimental Thermal and Fluid Science 71 (2016) 86-94.

17) Seo Young Kim, Byung Ha Kang and Jin-Ho Kim," Forced convection from materials in an asymmetrically heated channel", International Journal of Heat and Mass Transfer 44 (2001) 1451-1454.

18) Pradeep. M. Kamath, C. Balaji and S.P. Venkateshan, "Experimental investigation of flow assisted mixed convection in vertical channels", International Journal of Heat and Mass Transfer 54 (2011) 5231-5241.

19) R.B. Abernethy, R.P. Benedict, R.B. Dowdell, ASME Measurement Uncertainty,ASME Paper, 1983, 83-WA/FM-3. 